Vol. 4. No. 1. (290-311) Enero-Junio 2019

ISSN: 2539-1518

\title{
Modelo de transferencia por procesos propuesto por Margarita Amestoy de Sánchez para desarrollar la habilidad de observación
}

\author{
Elpidia Hernández Maldonado \\ elpidiahernandes@mail.uniatlantico.edu.co \\ Universidad del Atlántico \\ Meybin Pérez Padilla. \\ meybinperezpadilla@hotmail.com \\ Institución educativa distrital el Santuario
}

\section{Resumen}

El presente artículo ilustra un ejercicio de carácter cognitivo cuya finalidad es la de implementar el Modelo de Transferencia por Procesos, propuesto por la doctora Margarita Amestoy de Sánchez, con el objetivo de desarrollar la habilidad de observación en diferentes contextos. Esto se logró gracias a la adquisición de los fundamentos teóricos del modelo, el diseño y la aplicación de talleres que parten de un nivel de desarrollo básico de observación, hasta llegar a un nivel de mayor complejidad. Para realizar el diseño de los mismos, se tomaron insumos de los diferentes procesos del modelo y se asumieron como subprocesos, siendo cada uno de ellos un indicador del nivel de desarrollo de la destreza por taller. De acuerdo con la experiencia y la praxis obtenida durante la implementación, se concluye que con el MTP (Modelo de transferencia por procesos) se desarrolló la habilidad básica de observación.

\section{Palabras clave}

Habilidad de pensamiento, Modelo de Transferencia por Proceso, observación, Subprocesos.

Recibido 5/03/2019*Aceptado 17/05/2019 


\section{Introducción}

El análisis realizado por el Icfes (2010) sobre los resultados del estudio PISA 2009 y presentados por la OCDE (Organización para la Cooperación y el Desarrollo Económico) sugieren un problema que merece atención en el contexto colombiano, porque aunque la prueba mostró una moderada mejora en el desempeño de las competencias de los estudiantes, este aumento no es suficiente para garantizar las metas que el país se ha propuesto en materia de competitividad y equidad, ni tiene la velocidad requerida para alcanzar estándares de calidad internacional en educación como lo plantea la Revolución Educativa (2002). Según el análisis que realizó el ICFES (2010) sobre los resultados PISA 2009, Colombia tiene, respecto a las habilidades de lectura, niveles de desarrollo que son preocupantes a pesar de los avances observados desde 2006. Prácticamente la mitad de los estudiantes no alcanzó el nivel $2^{0}$, lo que significa que no tienen las habilidades básicas de lectura que les permitan participar de manera productiva en la sociedad moderna. Como consecuencia, estos jóvenes no podrían ingresar a la educación superior para enfrentar los retos que implican seguir aprendiendo a lo largo de sus vidas, lo que reduciría sustancialmente sus posibilidades de progreso individual, social y económico.

Por otra parte, menos del 1\% de los alumnos logró un desempeño sobresaliente en las tres áreas evaluadas, lo que también muestra una seria desventaja del país en cuanto a su capacidad de realizar innovaciones y de competir en igualdad de condiciones con otras regiones (ICFES, 2010, p. 42). Tomando como referencia lo anterior, se considera que un factor importante para elevar la calidad de la educación a nivel nacional lo constituye el cambio en la práctica pedagógica de los educadores (Revolución Educativa, 2002). Por tal razón, Margarita Amestoy de Sánchez propone en su programa Desarrollo de Habilidades del Pensamiento, el uso del Modelo de Transferencia por Procesos, cuyo objetivo es contribuir a satisfacer esta necesidad y desarrollar habilidades que propicien un aprendizaje más perdurable, significativo y de mayor aplicabilidad en la toma de decisiones y en la solución de problemas relacionados con las situaciones que el individuo se enfrenta en su interacción con el medio (Sánchez, 1991, p.5). Este Modelo de Transferencia por Procesos incluye el estudio de un conjunto de procesos que propician el desarrollo de diferentes tipos de estructuras cognitivas. Se trata de que el estudiante construya, sea por inducción o por deducción, los procedimientos de 
pensamiento que él utiliza progresivamente para adquirir conocimiento, administrar su aprendizaje y verificar su progreso (Sánchez, 1991, p.6). Ahora bien, este proyecto enfatiza en el proceso básico de observar, puesto que es la base para desarrollar las habilidades de pensamiento más avanzadas.

La realización de observaciones se halla presente en las prácticas de enseñanza de la mayoría de las carreras de formación profesional. Por ser tan habitual, se podría pensar que se trata de una práctica fácil que no requiere mayores conocimientos y habilidades. No obstante, las descripciones derivadas de esta actividad y expuestas por los estudiantes a través de informes escritos o comentarios orales en clase, dan cuenta que, por lo general, los propósitos iniciales que guiaron la actividad, lejos de conseguirse, se extravían ante la ausencia de orientaciones respecto a qué y cómo observar (Amieva, 2008, p. 1). Dada la importancia del desarrollo de esta habilidad en la formación de todo profesional, se considera importante centrar, incorporar y desarrollar de manera significativa las prácticas de observación a la enseñanza. Por consiguiente, a partir de la situación planteada se formula el siguiente interrogante: ¿Cómo se aplica el Modelo de Transferencia por procesos propuesto por Margarita Amestoy de Sánchez para desarrollar la habilidades de pensamiento, en particular la de observación?

La sociedad actual requiere seres humanos que se preocupen por ser más competitivos intelectualmente en diferentes contextos. Para lograrlo, es necesario de una educación integral (técnica, científica y humana) que permita al estudiante desarrollar su pensamiento con el objetivo de prepararlo para que interactúe de forma adecuada en los contextos personal y laboral. Pero, sobre todo, para que genere nuevos conocimientos de acuerdo con los cambios globales asociados a la tecnología, la proliferación de información y los avances científicos. Teniendo en cuenta lo anterior, durante los últimos años, la sociedad colombiana ha replanteado sus políticas educativas con el fin de alcanzar mejores condiciones de desarrollo social y económico que permita a los ciudadanos tener una mejor calidad de vida (Revolución Educativa, 2002) por medio de una educación crítica, científica, ética y tolerante, que brinde respuestas a las necesidades de cobertura, calidad, pertinencia laboral, capacitación técnica e investigación científica. El presente trabajo está encaminado hacia el desarrollo de las habilidades básicas de pensamiento, en particular la habilidad de observación. Para Margarita Amestoy de Sánchez (1991, p.29), la observación es una habilidad de pensamiento básico y primitivo, considerada como la clave o punto de partida para desarrollar las demás habilidades de 
pensamiento. Mediante la práctica de determinados ejercicios, se logra razonar de forma consciente, ordenada y sistemática potencializando el intelecto que tienen los seres humanos. En virtud de lo anterior, este proyecto responde a la necesidad de desarrollar una habilidad básica de pensamiento, de tal manera que se utilice intencionalmente el pensamiento y sus consecuentes procesos mentales. Así, de lo que se trata es de construir juicios razonables que se vean reflejados en la solución de problemas y en la toma de decisiones que permitan mejorar el rendimiento académico y la calidad de vida (Suárez, Cabrera, Roa \& Guzmán, 2007, p. 7).

El Modelo de Transferencia por Procesos incluye el estudio de un conjunto de procesos que propician el desarrollo de diferentes tipos de estructuras cognitivas. Es importante que el aprendiz construya por inducción o por deducción los procedimientos de pensamiento que él utiliza progresivamente para adquirir conocimiento, administrar su aprendizaje y verificar su progreso (Amestoy de Sánchez, 1991, p.6). Dada la importancia del desarrollo de la habilidad de observación en la formación de todo profesional, resulta significativo centrar, incorporar y desarrollar de manera ostensible las prácticas de observación en la enseñanza. Por tanto, a partir de la situación planteada se formuló el siguiente interrogante: ¿Cómo se aplica el Modelo de Transferencia por Procesos propuesto por Margarita Amestoy de Sánchez para desarrollar las habilidades de pensamiento, en particular la de observación? Para dar respuesta a este interrogante se estableció como objetivo general del proyecto: Implementar el Modelo de Transferencia por Procesos para desarrollar la habilidad básica de pensamiento de observación. Como objetivos específicos, uno, comprender los fundamentos teóricos del Modelo de Transferencia por Procesos para desarrollar la habilidad básica de pensamiento de observación; dos, diseñar actividades por medio del Modelo de Transferencia por Procesos para desarrollar la habilidad básica de pensamiento de observación y tres, aplicar el Modelo de Transferencia por Procesos con el fin de desarrollar la habilidad de observación.

\section{Marco de referencia}

Los referentes teóricos en que se basa este proyecto ayudan a comprender la relación (teórica y práctica) entre el Modelo de Transferencia por Procesos y la aplicabilidad del mismo en la enseñanza de destrezas básicas de pensamiento. De esta manera, nuestros referentes teóricos son: Jean Piaget (1986), por su teoría del desarrollo humano; Lev Vygotsky (1978,1987), por su teoría histórico sociocultural; Ángel Villarini 
(1991), por sus aportes acerca del desarrollo de las destrezas básicas de pensamiento y Margarita de Sánchez (1991), por sus investigaciones encaminadas al desarrollo de las habilidades básicas de pensamiento.

Piaget (1978) señala que la inteligencia consiste en la adquisición de un conjunto de estructuras o esquemas que en el transcurso de la vida se organizan de una forma determinada, implicando niveles de complejidad progresiva. Estas estructuras cambian debido a los procesos de asimilación y acomodación de la información del intelecto humano cuando interactúa con su medio social y natural. El proceso de asimilación consiste en la internalización de un objeto o un evento a una estructura comportamental y cognitiva preestablecida, mientras que la acomodación consiste en la modificación de la estructura cognitiva o del esquema comportamental para acoger nuevos objetos y eventos que hasta el momento eran desconocidos para el individuo. La adaptación está siempre presente en estos dos elementos básicos, que en algunos momentos buscan la estabilidad $\mathrm{y}$, en otros el cambio, permitiendo al sujeto aproximarse y lograr un ajuste dinámico con el medio (Villarini, 1997, p.42).

Margarita Amestoy de Sánchez (1991, p.139) menciona en su Modelo de Transferencia por Procesos que el pensamiento se adquiere por procesos que aumentan gradualmente sus niveles de complejidad y abstracción, constituyendo acciones o mecanismos mentales que el individuo utiliza en forma organizada y coordinada para adquirir y elaborar la información. Es decir, está implícito el proceso de asimilación, acomodación y adaptación, mencionado por Piaget y, por consiguiente, podemos decir que ambos modelos de pensamiento guardan cierto grado de interrelación. Es necesario precisar que el potencial de desarrollo humano no depende tanto de factores genéticos o de la maduración (como lo plantea Piaget), sino de la ayuda de otras personas de un nivel más alto de desarrollo y de la interacción del organismo con su medio ambiente, es decir, la cultura. Esa ayuda tiene como objetivo el desarrollo cognitivo del individuo a través de la capacidad para observar, solucionar problemas y construir cosas que por sí solo no podría (Vygotsky, 1987). La teoría socio-cultural fue construida sobre la premisa de que el desarrollo intelectual no puede comprenderse sin una referencia al mundo social del niño. El desarrollo individual se entiende sobre la base de las raíces sociales de los instrumentos que el niño utiliza para pensar. El contexto socio-cultural llega a ser accesible al individuo a través de la interacción social con otros miembros de la sociedad que conocen mejor el manejo y las destrezas de los instrumentos intelectuales. La interacción social se convierte en el motor del desarrollo e introduce el concepto de 
“Zona de Desarrollo Próximo", que se concibe como la distancia entre el nivel real de desarrollo y el nivel de desarrollo potencial. Para determinar este concepto, en términos propuestos por Vygotsky, se deben tener presente dos aspectos, a saber: la importancia del contexto social y la capacidad de imitación (Villarini, 2001).

La teoría de Vygotsky fue importante para este proyecto porque la interacción socio-cultural mediada por el uso de herramientas permite evolucionar hacia formas cada vez más complejas y perfectas mediante un proceso psíquico de reestructuración y reorganización cualitativa. En este caso, el Modelo de Transferencia por Procesos pretende que la persona, al conocer los procesos y los procedimientos, desarrolle habilidades de pensamiento (observación) que aplicará y transferirá -de manera consciente- a diferentes contextos de su vida diaria (de Sánchez,2001).Teniendo en cuenta las palabras de Villarini cuando afirma que "el pensamiento es la capacidad que tiene el ser humano para construir una representación e interpretación mental significativa de su relación con el mundo" (Villarini, 1991, p.10), podemos decir que el pensamiento es un proceso que se inicia en la recepción (insumo) de la realidad y ésta se capta por medio de los sentidos, así son transformados en percepciones o información que se encuentran archivadas en la memoria y desde esa primera aprehensión se activan procesos cognitivos condicionados por los conceptos y actitudes del pensamiento. Según Villarini, la enseñanza orientada al desarrollo de pensamiento (1991, pp. 58-59) tiene tres componentes que le dan su particular estructura, a saber:

a) Concepto: Regla que orienta la actitud de la destreza.

b) Destreza: Actividad básica del pensamiento en su procesamiento de información.

c) Actitud: Disposición que facilita la ejecución de la destreza en la aplicación del concepto.

El pensamiento es la capacidad o competencia para procesar información y construir conocimiento, combinando representaciones, operaciones y actitudes mentales en forma automática, sistemática, creativa o crítica para producir creencias y conocimientos, plantear problemas y buscar posibles soluciones, tomar decisiones, comunicarse e interactuar con otros y establecer metas y medios para su logro (Villarini, 1997). Villarini define la destreza de pensamiento como diversas actividades u operaciones que la mente lleva a cabo sobre la información para reestructurarla u organizarla y producir un nuevo 
conocimiento. Las destrezas son procedimientos o "pasos" que se ejecutan sobre la información.

El autor asegura que existen dos tipos de destrezas:

1. Las destrezas básicas, como aquellas que se limitan a un procesamiento simple de los estímulos o información, como por ejemplo: observar, recordar, comparar con información previa. Aunque simples, estas destrezas son necesarias (Villarini, 1991, p. 30). Las destrezas complejas o superiores no podrían emplearse si primero las básicas no recibieran e interpretaran superficialmente la información.

2. Las destrezas avanzadas o complejas procesan la información a un nivel de mayor profundidad o complejidad (relaciones y conexiones). El análisis, la evaluación y la solución de problemas son procesos más complejos pues requieren de la combinación de varias destrezas y una multiplicidad de conceptos. (Villarini, 1991, p. 30).

Villarini define la destreza básica de observar como la acción de fijar la atención en los tributos o características de los objetos y describirlas mediante el uso del conocimiento previamente adquirido. Esta habilidad se trabaja de la siguiente manera:

a) Establecer un propósito: Percibir un objeto a través de uno o varios canales sensoriales.

b) Fijar la atención en las características del objeto.

c) Identificar las características del objeto mediante el uso de conocimiento previo (Villarini, 2001).

La teoría de Villarini ha sido importante para este proyecto dado que el desarrollo de la destreza de pensamiento de observar, es un requisito indispensable para que el aprendiz obtenga el éxito académico y el máximo provecho de las asignaturas. Esto resulta ser un proceso significativo porque mediante el desarrollo de la capacidad del pensamiento se puede preparar al aprendiz para que sea mejor ser humano, mejor ciudadano, mejor trabajador y mejor profesional (Villarini, 1991, p.3).

Por su parte, Margarita Amestoy de Sánchez (2002, p. 142), con su Modelo de Transferencia por Procesos, menciona que por medio del desarrollo de habilidades de pensamiento se obtiene éxito académico, dando lugar a la generación de las estructuras 
cognitivas indispensables para construir, extender y transferir el conocimiento a variedad de ámbitos, situaciones y áreas de saber con el objeto de interactuar satisfactoriamente con el medio ambiente, y en general a controlar el desarrollo personal (intelectual y emocional). Según esta autora, pensar es un proceso complejo que no está claramente explicado; sin embargo, con fines prácticos y de investigación, se elaboran modelos hipotéticos explicativos que pueden ser conceptuales u operacionales. Pensar de acuerdo con el modelo de procesos, es un acto que involucra un constructo complejo y abstracto (el pensamiento), y como tal está constituido por otros constructos también de diferentes grados de complejidad y abstracción (Sánchez, 2002, p.133). Los procesos de pensamiento constituyen acciones o mecanismos mentales que realiza la persona en forma organizada y coordinada para adquirir y elaborar la información. La enseñanza de procesos de pensamiento abarca una actividad destinada a desarrollar habilidades de pensamiento, es decir, hábitos para aplicar, en forma natural y espontánea, los procesos en cualquier acto físico o mental en el cual sean requeridos (Sánchez, 2002. p, 29). En su texto Desarrollo de habilidades de pensamiento (1991), la autora señala que durante los últimos años educadores y psicólogos han observado que el desempeño intelectual de los estudiantes ha disminuido (1991, p. 5). La investigación demuestra que las dificultades observadas aumentan conforme se alcanza mayor grado de escolaridad, se vuelve apremiante en la universidad y además está presente en el desempeño profesional y familiar. Se ha comprobado, asimismo, que muchas de estas dificultades tienen relación con la carencia de habilidades para procesar la información y repercuten en el desarrollo de esquemas que faciliten el almacenamiento, la recuperación y el uso apropiado de los conocimientos. Por consiguiente, se buscan posibilidades que ayuden a corregir dicha situación, y Sánchez contribuye a satisfacer esta necesidad propiciando el desarrollo de habilidades para lograr un aprendizaje más perdurable, significativo y de mayor aplicabilidad en la toma de decisiones y la solución de problemas relacionados con las situaciones a las que el individuo se enfrenta en su interacción con el medio. En este sentido, afirma:

[...] la práctica de procedimientos, bajo condiciones controladas, genera las habilidades de pensamiento. El proceso existe por sí mismo, independientemente de la persona que lo ejecuta, el procedimiento proviene de la operacionalización del proceso y la habilidad es una facultad de la persona, cuyo desarrollo requiere de un aprendizaje sistemático y deliberado (Sánchez, 2002, p. 139). 
Ella agrupa y ordena los procesos de pensamiento de acuerdo con sus niveles de complejidad y abstracción: Procesos básicos, constituidos por seis operaciones elementales: observación, comparación, relación, clasificación simple, ordenamiento y clasificación jerárquica. $\mathrm{Y}$ tres procesos integradores: análisis, síntesis y evaluación. Dentro de estas habilidades de pensamiento, el Modelo de Transferencia por Procesos define la observación como el proceso que consiste en fijar la atención en un objeto o situación para identificar sus características. Los procedimientos para realizar la observación son:

a) Definir el propósito de la observación.

b) Enumerar las características del objeto o situación tomando en cuenta el propósito, con el fin de transformarlo en una habilidad que permita aplicar el conocimiento procedimental en diferentes contextos o situaciones.

La didáctica basada en este Modelo de Transferencia por Procesos es el pilar de este proyecto, ya que se constituye en una estrategia de enseñanza- aprendizaje integral apoyada en la reestructuración cognitiva del sujeto, tomando como base la reformulación consciente y deliberada de los esquemas de procesamiento que resulten requeridos para lograr que las personas piensen con claridad y desarrollen los modelos de procesamiento mental utilizándolos con eficacia y efectividad. De esta manera, podrán aprender y resolver los problemas que confronten en cualquier ámbito en el que tengan que desempeñarse (Sánchez, 2002, p. 143).

\section{El modelo de enseñanza: transferencia por procesos}

En el Modelo de Transferencia por Procesos que desarrolló Sánchez, los procesos son conceptos y cada proceso tiene un significado que lleva implícito una acción que lo caracteriza. Ésta es ejecutada siguiendo el procedimiento que corresponde y la práctica de procedimientos bajo condiciones controladas genera las habilidades de pensamiento (2002). A continuación, se observa un esquema que ilustra la relación entre proceso, procedimiento y habilidad y las categorías que constituyen el Modelo de Transferencia por procesos, además de los insumos que se requieren para su desarrollo y funcionalidad (Gráfico 1). 


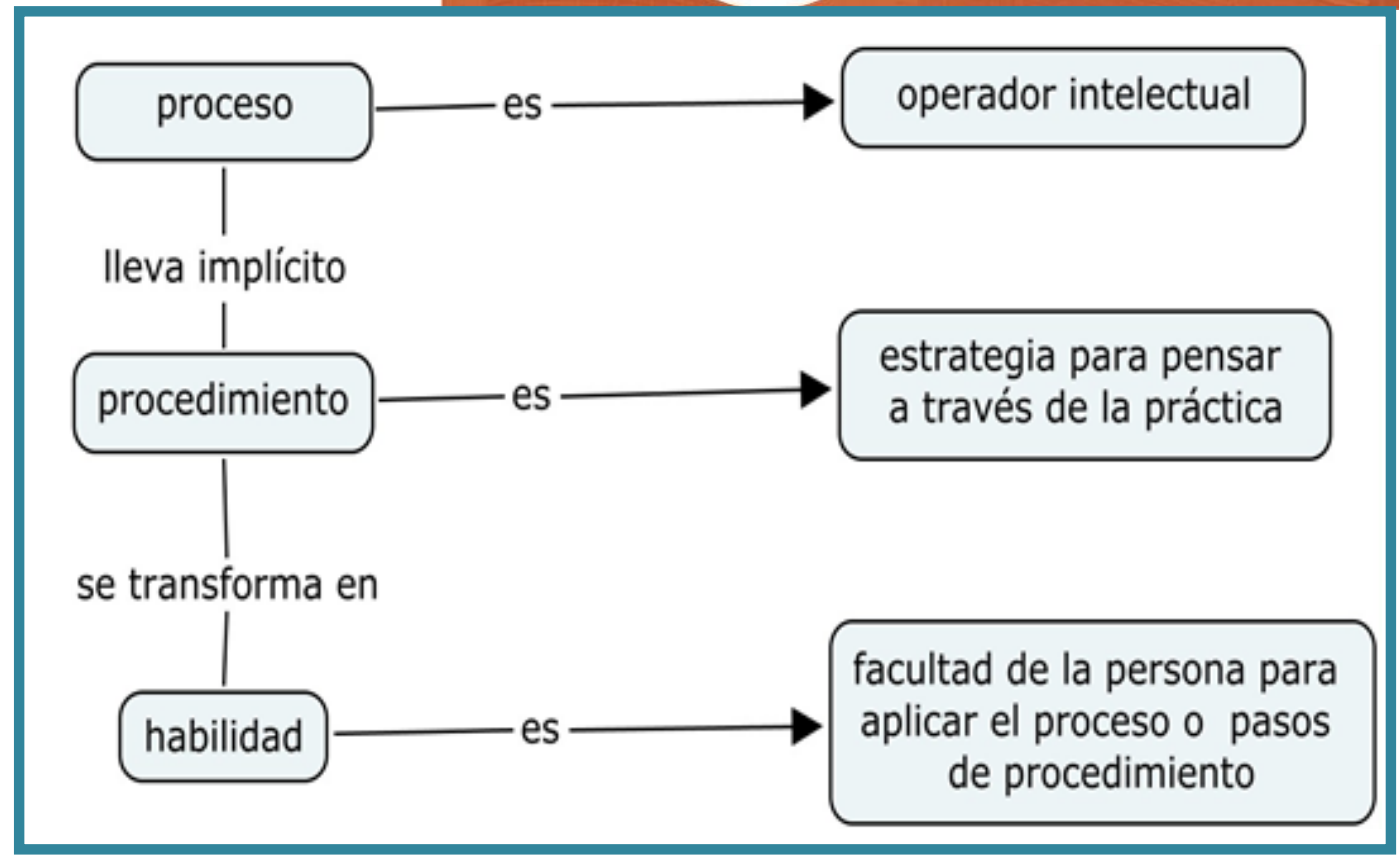

Gráfico 1. Modelo de enseñanza de transferencia por procesos (Amestoy de Sánchez, 2002).

De igual manera, observemos un cuadro comparativo acerca de las teorías usadas en este texto (Cuadro 1).

Cuadro 1. Comparativo de las teorías de los referentes teóricos usados en el presente artículo

\begin{tabular}{|c|c|c|c|c|}
\hline & $\begin{array}{l}\text { MARGARITA } \\
\text { AMESTOY DE } \\
\text { SÁNCHEZ }\end{array}$ & ÁNGEL VILLARINI & PIAGET & VYGOTSKY \\
\hline PENSAMIENTO & $\begin{array}{l}\text { El pensamiento } \\
\text { es una habilidad } \\
\text { que se puede } \\
\text { desarrollar y la } \\
\text { mayor parte de } \\
\text { esto ocurre en la } \\
\text { etapa de la } \\
\text { percepción, } \\
\text { cuando el sujeto } \\
\text { reacciona ante } \\
\text { los estímulos y } \\
\text { responde según } \\
\text { percibe su } \\
\text { mundo. }\end{array}$ & $\begin{array}{l}\text { El pensamiento es la } \\
\text { capacidad r o } \\
\text { competencia para } \\
\text { procesar información } \\
\text { y construir } \\
\text { conocimiento } \\
\text { combinando } \\
\text { representaciones, } \\
\text { operaciones } \\
\text { actitudes mentales en } \\
\text { forma automática, } \\
\text { sistemática, creativa o } \\
\text { crítica, para producir } \\
\text { creencias } \\
\text { conocimientos, y } \\
\text { plantear problemas y } \\
\text { posibles soluciones, } \\
\text { tomar decisiones, } \\
\text { comunicarse } \\
\text { interactuar con otros, } \\
\text { así como establecer } \\
\text { metas y medios para }\end{array}$ & $\begin{array}{l}\text { El pensamiento } \\
\text { aparece cuando la } \\
\text { función simbólica se } \\
\text { comienza a } \\
\text { desarrollar, aunque } \\
\text { para el autor los } \\
\text { esquemas de acción, } \\
\text { los cuales serán la } \\
\text { base para un posterior } \\
\text { aprendizaje del } \\
\text { lenguaje, comienzan a } \\
\text { construirse en el } \\
\text { periodo sensorio- } \\
\text { motor } \\
\text { Existen cinco niveles } \\
\text { de pensamiento } \\
\text { consecuenciales y } \\
\text { evolutivos respecto al } \\
\text { anterior: } \\
\text { 1.Pensamiento } \\
\text { simbólico }\end{array}$ & $\begin{array}{l}\text { El pensamiento es } \\
\text { una construcción } \\
\text { social que se hace } \\
\text { posible a través } \\
\text { de la interacción } \\
\text { con el medio que } \\
\text { nos rodea, para } \\
\text { luego } \\
\text { procesado ser } \\
\text { través de un } \\
\text { lenguaje } \\
\text { finalmente, } \\
\text { constituir } \\
\text { lenguaje un } \\
\text { interiorizado. }\end{array}$ \\
\hline
\end{tabular}




\begin{tabular}{|c|c|c|c|c|}
\hline & & su logro. & $\begin{array}{l}\text { 2.Pensamiento } \\
\text { preconceptual } \\
\text { 3.Pensamiento } \\
\text { intuitivo } \\
\text { 4.Pensamiento lógico } \\
\text { 5.Pensamiento formal }\end{array}$ & \\
\hline OBSERVACIÓN & $\begin{array}{l}\text { Proceso que } \\
\text { consiste en fijar la } \\
\text { atención en un } \\
\text { objeto o situación } \\
\text { para identificar } \\
\text { sus } \\
\text { características. } \\
\text { La identificación } \\
\text { ocurre en dos } \\
\text { etapas: } \\
\text { primera, la } \\
\text { concreta, y la } \\
\text { segunda, } \\
\text { abstracta. }\end{array}$ & $\begin{array}{l}\text { Fijar la atención en los } \\
\text { atributos o } \\
\text { características de los } \\
\text { objetos y describirlos } \\
\text { mediante el uso del } \\
\text { conocimiento } \\
\text { previamente } \\
\text { adquirido. }\end{array}$ & $\begin{array}{l}\text { Se da en las } \\
\text { diferentes etapas } \\
\text { cognitivas del ser } \\
\text { humano, adquiriendo } \\
\text { mayor destreza a } \\
\text { medida que avanza a } \\
\text { la siguiente etapa. }\end{array}$ & $\begin{array}{l}\text { Considera que el } \\
\text { niño aprende a } \\
\text { través de la } \\
\text { interacción con su } \\
\text { medio ambiente y } \\
\text { otras personas. }\end{array}$ \\
\hline $\begin{array}{c}\text { INFERENCIAS } \\
\text { DE LAS } \\
\text { TEORIAS }\end{array}$ & $\begin{array}{l}\text { La observación } \\
\text { es el medio por el } \\
\text { cual se reacciona } \\
\text { a los estímulos, y, } \\
\text { por ende, se } \\
\text { estimula el } \\
\text { pensamiento. }\end{array}$ & $\begin{array}{l}\text { El pensamiento es la } \\
\text { manera en que el ser } \\
\text { humano se relaciona } \\
\text { con el mundo: para } \\
\text { relacionarse con él } \\
\text { debe percibirlo a } \\
\text { través de la } \\
\text { observación. }\end{array}$ & $\begin{array}{l}\text { El ser humano posee } \\
\text { desde su nacimiento } \\
\text { un potencial de } \\
\text { desarrollo } \\
\text { neurocerebral que, } \\
\text { dadas ciertas } \\
\text { estimulaciones de } \\
\text { condición social, se } \\
\text { transforma en } \\
\text { capacidad de } \\
\text { pensamiento. }\end{array}$ & $\begin{array}{l}\text { El pensamiento es } \\
\text { una construcción } \\
\text { social que se hace } \\
\text { posible a través } \\
\text { de la interacción } \\
\text { con el medio que } \\
\text { nos rodea, y esta } \\
\text { es captada a } \\
\text { través de los } \\
\text { sentidos, y, por } \\
\text { tanto, mediante la } \\
\text { observación. }\end{array}$ \\
\hline
\end{tabular}

\section{Antecedentes}

Desarrollar una habilidad implica la superación de la siguiente secuencia de etapas: conocimiento y comprensión de la operación mental que define el proceso; concientización de los pasos que conforman la definición operacional del proceso; aplicación y transferencia del proceso a variedad de situaciones y contextos; generalización de la aplicación del procedimiento; y evaluación y mejora continua del procedimiento. Para lograr la habilidad de aplicar el proceso de manera efectiva, es necesario practicarlo hasta lograr el hábito de utilizarlo en forma natural y espontánea, en variedad de situaciones y contextos, adaptándolo de acuerdo a los requerimientos de la tarea (Sánchez, 2002). La enseñanza basada en procesos propone la aplicación de los procesos como instrumentos para dos propósitos: a) el manejo del conocimiento, y b) el 
diseño de una didáctica que conduzca al logro del aprendizaje. Los procesos permiten seleccionar y organizar los conocimientos que se van a impartir, conceptuar y operacionalizar mediante una metodología de enseñanza efectiva, basada en un modelo de aprendizaje activo, significativo y centrado en el constructivismo cognoscitivo, y dirigido al desarrollo de la potencialidad de las personas, para aprender y aprender a aprender. Por esta razón, se han realizado estudios que implementan este modelo, entre ellos, se destaca Estrategias didácticas para activar el desarrollo de los procesos de pensamiento aplicado en el nivel pre-escolar (Cañizales, 2004), el cual tuvo como objetivo determinar la efectividad del Modelo de Transferencia por Procesos en la aplicación de estrategias didácticas que activan el pensamiento del niño, y que arrojó como resultado que el modelo funciona para activar dichos procesos, entre ellos la observación. Así mismo, se encuentra el estudio Concepciones docentes sobre la promoción del desarrollo de la destreza "observar" y su evidencia en la práctica pedagógica (Escalante, 2009), cuyo propósito fue establecer si las concepciones sobre promoción del desarrollo de la destreza de pensamiento "observar" de docentes del grado transición (pre-escolar) son evidentes en su práctica pedagógica. Sus resultados demostraron que las concepciones de los docentes evidencian la importancia del desarrollo de la destreza de pensamiento "observar" como base para el desarrollo de destrezas de pensamiento más complejas y facilitadoras de otros aprendizajes.

De igual forma, está la investigación sobre el desarrollo y la enseñanza de las habilidades de pensamiento, cuya presentación propone y justifica un modelo integrado de investigación y desarrollo aplicable a la construcción, implantación y evaluación de proyectos para la enseñanza y transferencia de habilidades de pensamiento (VI Congreso Nacional de Investigación Educativa, 2002). También, podemos encontrar el proyecto Validación de una prueba de habilidades de pensamiento para alumnos de cuarto y quinto de secundaria y primer año de universidad, el cual da a conocer los resultados de un estudio psicométrico de una prueba elaborada con el fin de evaluar cuantitativa y cualitativamente los procesos cognitivos básicos y superiores del pensamiento mediante la comprensión lectora. Los dominios que se tuvieron en cuenta en este instrumento son el cognitivo y el lingüístico, teniendo como marco teórico de referencia la Teoría de la Modificabilidad Cognitiva Estructural de Reuven Feuerstein, complementada con aspectos de la teoría basada en procesos, de Sánchez. A partir de esto, se concluyó que la prueba de habilidades de pensamiento ha demostrado tener confiabilidad, tanto en su 
totalidad como en los diferentes ítems que la conforman por consistencia interna (Tapia \&Luna, 2010).

Igualmente, podemos resaltar entre los diferentes estudios Habilidades básicas del pensamiento y competencia para aprender a aprender de Justo de la Rosa (2009), donde se evidencia que aprender a aprender implica la conciencia, gestión y control de las propias capacidades y emociones desde un sentimiento de competencia que permite el desarrollo de las habilidades básicas del pensamiento. Esto hace que la persona tome conciencia de sus capacidades y utilice sus habilidades para planificar, desarrollar y evaluar el propio aprendizaje, y además se sienta motivada y disponga de la confianza en sí misma, necesaria para utilizar el aprendizaje de forma autónoma y eficaz en los distintos contextos.

\section{Diseño metodológico}

La metodología que se desarrolló para este estudio, estuvo enfocada en implementar el Modelo de Transferencia por Procesos propuesto por Margarita Amestoy de Sánchez (1991) para desarrollar la habilidad básica de observación. Además de conocer los fundamentos teóricos y prácticos, tanto del modelo como de la destreza de pensamiento en estudio, se adquirió la experticia en su aplicación no solo en la dinámica misma del proceso llevado a cabo, sino también en la posibilidad de transferir dicho conocimiento a diferentes contextos de la vida cotidiana. Para desarrollar esta experticia fue necesario comprender que los fundamentos teóricos del Modelo de Transferencia por Procesos están encaminados a dos propósitos, esto es: el manejo del conocimiento y el diseño de una didáctica que condujese al aprendizaje de una destreza básica de pensamiento (observar).

Si bien el estudio se orientó hacia la comprensión y aplicación del Modelo de Transferencia por Procesos para un objeto de aprendizaje específico (una destreza básica de pensamiento), lo cierto es que de acuerdo con la literatura revisada, dicha propuesta puede ser trasladada a otros contextos y propósitos de enseñanza (Sánchez, 1991, p.6). Gracias al Modelo de Transferencia por Procesos, se seleccionaron y organizaron los conocimientos tomados como pretexto de enseñanza, de tal manera que el dominio conceptual y operacional de la destreza de pensamiento y de observación se pudo estructurar dentro de un modelo orientado hacia el aprendizaje activo, significativo y 
centrado en el constructivismo cognoscitivo, y dirigido al desarrollo de la potencialidad de las personas (2002, p. 142). Los criterios tomados en cuenta para la comprensión y el diseño de actividades aplicando el Modelo de Transferencia por Procesos, fueron los siguientes:

a. La intencionalidad del acto mental y de la actividad mediante la cual se dirige el uso de la capacidad intelectual del individuo.

b. La concientización del acto mental involucrado en el proceso.

c. El enfoque del sistema como instrumento de pensamiento, como producto de la metodología de procesos y como fuente de retroalimentación y de optimización del acto mental.

d. La participación activa del aprendiz en la construcción y refinamiento del conocimiento.

e. Aplicación de un conjunto de estrategias didácticas para estimular el aprendizaje y diagnosticar el progreso de los alumnos y el papel del docente como mediador del proceso de enseñanza aprendizaje (Sánchez, 2002, p. 142).

A partir de estos criterios, se elaboró el proyecto encaminado a desarrollar la destreza básica de observación, teniendo en cuenta las siguientes fases constitutivas del Modelo de Transferencia por Procesos (Sánchez, 2002, p. 139): Justificación, objetivos, introducción, preguntas de desarrollo, evaluación, proceso de mejoramiento y verificación del proceso estudiado en el taller a través de preguntas específicas. Con el desarrollo, aplicación y sistematización de este proyecto se ilustra un ejercicio de carácter cognitivo para adquirir la experticia en el diseño, aplicación y transmisión idónea del Modelo de Transferencia por Procesos para desarrollar la habilidad de observación en diferentes contextos. Esta experticia se desarrolló con el diseño y aplicación de talleres, partiendo de un nivel de desarrollo básico de observación, hasta llegar a un nivel de mayor complejidad. Para realizar el diseño de los mismos, se tomaron insumos de los diferentes procesos del modelo y se asumieron como subprocesos, siendo cada uno de ellos un indicador del nivel de desarrollo de la destreza por taller. En tal sentido, de un taller a otro se verificó que se hubiese gestado un "proceso de anclaje"1, es decir, se

\footnotetext{
${ }^{1}$ En la síntesis de diversas teorías cognitivas, el concepto de "anclaje" alude al proceso de asociar una respuesta interna con algún disparador externo o interno a fin de que la respuesta pueda ser rápida y algunas veces secretamente accesible. El anclaje es un proceso que, en la superficie, es similar a la técnica "condicionante" usada por Pavlov (1926). El proceso de anclaje tiene como objetivo solidificar y transferir
} 
definieron unas pautas o criterios de verificación del desarrollo de la destreza de observación a partir de los subprocesos empleados para la aplicación del Modelo de Transferencia por Procesos. Gracias a la conceptualización propuesta para la aplicación del modelo, se llegó a un ejercicio de comparación, relación y diferenciación entre las propuestas de Sánchez (1991) y la de Villarini (1991), en función de los propósitos y alcances expuestos por estos teóricos en relación con el desarrollo específico de la destreza de pensamiento propuesta para la implementación de este estudio.

De igual manera, se definieron fases para estructurar la forma en que se ejecutó la aplicación para cada uno de los subprocesos que determinaron el desarrollo de la destreza básica de observación. Previo a la aplicación de los talleres, se hizo un trabajo de contrastación por parte de pares académicos, para definir el perfil de los evaluadores a partir de las siguientes características:

Docentes en ejercicio en un área de formación específica al interior de una institución educativa media vocacional o de Educación Superior, con una experiencia de entre 10 y 15 años y con un nivel de formación de Especialista o Magister, cuyo título estuviese directamente relacionado con educación. Los criterios de evaluación que los pares tuvieron en cuenta para realizar su evaluación y retroalimentación de los talleres fueron el de la claridad y la de precisión. El proceso de los talleres se registró y evaluó a través de unas rúbricas de evaluación y verificación del proceso de aprendizaje orientado a entender el proceso de comprensión, incorporación y dominio desde el punto de vista de la experticia. Esto se documentó en una rúbrica evaluativa del proceso de aplicación que parte del nivel de desempeño bajo hasta llegar al desempeño superior, permitiendo a los participantes de este proyecto comprender las fases y criterios que posee cada una de las fases del Modelo de Transferencia por Procesos en cada uno de los subprocesos de la observación y el nivel de complejidad de observación que desarrolló.

experiencias aprendidas. Una gran cantidad de aprendizaje guarda relación con condicionamiento, y el condicionamiento guarda relación con el tipo de estímulos que se asocian a las reacciones. (Dilts, 2008) 


\section{Fases de la investigación}

\begin{tabular}{|c|c|c|c|}
\hline FASE & ACTAS & № DE HORAS TRABAJADAS & ACTIVIDADES DESARROLLADAS \\
\hline $\begin{array}{c}\text { Fundamentos } \\
\text { teóricos }\end{array}$ & $1-11$ & $\begin{array}{l}194 \text { horas trabajadas } \\
\text { individualmente }\end{array}$ & $\begin{array}{l}\text { *Lecturas acerca del tema. } \\
\text { *Esquematización de la teoría por medio de mapas } \\
\text { conceptuales. } \\
\text { *Cuadro comparativo de las teorías estudiadas }\end{array}$ \\
\hline $\begin{array}{l}\text { Diseño de } \\
\text { rúbricas y } \\
\text { talleres }\end{array}$ & $12-18$ & $\begin{array}{l}176 \quad \text { horas } \\
\text { individualmente }\end{array}$ & $\begin{array}{l}\text { *Rúbrica de las fases y criterios del MTP. (40 horas) } \\
\text { *Instrumento de evaluación de pares.(11horas ) } \\
\text { *Matriz de evaluación y autoevaluación del proceso.(40 } \\
\text { horas) } \\
\text { *Rúbrica de evaluación y autoevaluación del proceso de } \\
\text { aplicación.(40 horas) } \\
\text { *Talleres (45 horas) }\end{array}$ \\
\hline $\begin{array}{l}\text { Aplicación de } \\
\text { rúbricas y } \\
\text { talleres }\end{array}$ & $19-25$ & 116 horas de trabajo & $\begin{array}{l}\text { *Instrumento de evaluación de pares. (4 horas) } \\
\text { *Talleres. ( } 48 \mathrm{hrs} \text { ) } \\
\text { *Transcripción de los talleres. ( } 32 \text { horas) } \\
\text { *Rúbrica de evaluación y autoevaluación del proceso de } \\
\text { aplicación (32 horas) }\end{array}$ \\
\hline
\end{tabular}

\section{Resultados}

Al finalizar las fases de este proyecto, se obtuvo como resultado la comprensión de los fundamentos teóricos del Modelo de Transferencia por Procesos (MTP) y de la destreza básica de observación por medio de consultas de la literatura y el trabajo de campo. Las fuentes bibliográficas consultadas fueron Vygostky (1978, 1979, 1987, 1995), Piaget (1976, 1978, 1986), Villarini (1991,1997, 2001, 2006) y Sánchez (1991,1992, 1999, 2002). Para tal efecto, se tomó a Vygotsky y a Piaget, por ser los pioneros en la construcción teórica y práctica de cómo se estructuran las teorías sobre la cognición humana. Se escogió a Villarini por su desarrollo teórico sobre el concepto de destrezas de pensamiento y a Sánchez por su propuesta acerca del Modelo de Transferencia por Procesos y la destreza básica de observación. 
La adquisición de la teoría permitió al autor del presente artículo comprender, a través del diseño de una rúbrica, los criterios que posee cada una de las fases del Modelo de Transferencia por Procesos (de aquí en adelante lo llamaremos MTP), según el subproceso a desarrollar. Su diseño se realizó en 40 horas de trabajo para, posteriormente, crear con eficacia y objetividad, en 11 horas de trabajo, un instrumento de evaluación de pares académicos que validaran la claridad y precisión de los criterios de cada uno de los subprocesos planteados en el proyecto. De igual manera, se diseñó en 40 horas una rúbrica de evaluación y verificación del proceso de aplicación con el objetivo de conocer los niveles de desempeño que debe alcanzar el aprendiz, teniendo en cuenta los indicadores de logros de cada uno de los subprocesos. Seguidamente, utilizando como referente la rúbrica anterior, se diseñó, en 40 horas, una matriz de evaluación y autoevaluación del proceso de aplicación. Y, finalmente, el diseño de talleres implicó 45 horas de trabajo. La evaluación realizada por los pares académicos implicó dos horas de trabajo para un total de 4 horas.

Con el objetivo de confirmar el aumento en el nivel de complejidad de la observación, se realizó la transcripción de los talleres en 32 horas de trabajo, 16 horas para los talleres de cada aprendiz. Finalmente, en 32 horas de trabajo, se conceptualizaron y descubrieron los niveles de desempeño en la habilidad de observación en la matriz de evaluación y autoevaluación dispuesta para verificar el proceso de aplicación. Con la aplicación de estos talleres, se pudo obtener como resultado que el Modelo de Transferencia por Proceso sí es pertinente para desarrollar la observación de objetos o situaciones en diferentes contextos, puesto que el modelo permitió a los aprendices reconocer las fases y criterios que posee el MTP en cada uno de los subprocesos de la observación y el nivel de complejidad de observación que desarrolló. La aplicación permitió la sistematización del MTP, por el uso reiterativo de cada fase que integran este modelo, partiendo de un nivel de observación básico hasta llegar a un nivel de observación complejo, enfocado en la relación de características, consistente en establecer un nexo entre dos características de un objeto o situación referidas a una misma variable (Amestoy, 1991). A continuación, se describe el proceso de complejidad en la adquisición de la destreza básica de observación: 
- Observación, observación directa, observación indirecta, observación de características, observación de semejanzas, observación de diferencias, observación enfocada en la comparación y observación enfocada en la relación.

Es también factible anotar que, para que haya un nivel de observación básico, son necesarios dos procedimientos: primero, definir el propósito de la observación y sus características, ya que en la observación directa e indirecta los procedimientos aumentan su nivel de complejidad porque es necesario definir el propósito de la observación y reconocer si el objeto o situación observada se dio de forma directa o indirecta. Segundo, conocer si se estableció contacto a través de la observación personal con el objeto o situación observada o si la observación se logró mediante conversaciones con otras personas, lecturas de libros o información suministrada por medios masivos de comunicación (Amestoy, 1991). Posteriormente, en la observación de características se define el propósito de la observación y además se realizan preguntas orientadas a identificar las características de los objetos o situaciones con el fin de formular la descripción de forma organizada y coherente. En la observación de semejanzas y la observación de diferencias se define el propósito de la observación y se identifican las variables de interés, esto es, un tipo de característica que permita organizar las observaciones, ya sea identificando las semejanzas o las diferencias con el fin de elaborar la lista de diferencias o semejanzas de los objetos o situaciones. Por último, verificar el producto obtenido. En la observación enfocada en la comparación, el nivel de complejidad se presenta en que no solo se define el propósito de la observación y se identifican las variables que definen el propósito, sino que se establecen semejanzas y diferencias entre las características de dos objetos o situaciones, considerando dicha característica independientemente, es decir, se trata de identificar variable por variable, que los pares de objetos o situaciones que se compara sean semejantes o diferentes entre sí para finalizar con la verificación del producto último. Finalmente, la observación llegó a su mayor nivel de complejidad cuando se definieron e identificaron las variables que precisan el propósito y las características semejantes y diferentes, correspondientes a cada variable, con el propósito de establecer interrelación entre dos características de un objeto o situación referidas a una misma variable, formular las relaciones y revisar el producto final (Amestoy, 1991). Su importancia radica en que se llega a un paso más del procesamiento de la información puesto que se consideran pares de características de una 
misma variable provenientes de la comparación y se conectan mediante una proposición que establece un nexo entre ellas.

\section{Conclusiones y recomendaciones}

A partir de que la implementación del Modelo de Transferencia por Procesos propuesto por Margarita Amestoy de Sánchez es pertinente para desarrollar la habilidad de observación, es recomendable:

a. Emplear las matrices de este proyecto en aulas de clase, orientadas a desarrollar la habilidad básica de observación por medio del MTP.

b. Realizar actividades de aplicación teniendo como punto de partida los diseños propuestos en este proyecto dirigidas a alcanzar el desarrollo de la habilidad de observación

c. Ser muy concreto en el proceso a desarrollar, abarcando un número limitado, de tal manera que pueda, además de hacerse experto, llevarlas a cabo. No es conveniente abarcar demasiado.

Con el propósito de enriquecer este estudio formativo, surgen los siguientes interrogantes:

1. ¿Cómo se puede aplicar el MTP para desarrollar la habilidad de observación en aulas de clase con grupos de estudiantes, donde el seguimiento personalizado es prácticamente inviable (se piensa en aulas de clase con un número superior a 20 estudiantes)?

2. ¿Qué tipo de estudios comparativos, tanto cuantitativos como cualitativos, se pueden desarrollar con el fin de analizar y comprender el impacto cognitivo del MTP para desarrollar la habilidad de observación con otra estrategia de enseñanza?

3. ¿Es posible ampliar y profundizar los alcances del MTP para desarrollar la habilidad de observación en diferentes áreas del conocimiento?

4. Al implementar el MTP para desarrollar la habilidad de observación, ¿es posible mejorar las relaciones en grupos de trabajo cuando se presentan problemas o diferencias? 
5. ¿Es posible generar una plataforma de formación y gestión para la aplicación del MTP y así lograr un desarrollo de la habilidad de observación en los maestros que se están formando? 


\section{Referencias bibliográficas}

Amestoy de Sánchez, M. (1991). Desarrollo de habilidades de pensamiento. México, D. F.: Trillas.

Amestoy de Sánchez, M. (2002). "La investigación sobre el desarrollo y la enseñanza de las habilidades de pensamiento". Revista electrónica de Investigación Educativa 4, (1). Consultado el 20 de junio de 2010 en: http://redie.uabc.mx/vol4no1/contenidoamestoy.html

Cañizales, J. (2004). Estrategias didácticas para activar el desarrollo de los procesos de pensamiento en el preescolar. Investigación y postgrados V. 19 N. 2. Consultado el 10 de noviembre de 2010 en:http://www.scielo.org.ve/scielo.php?pid=S131600872004000200008\&script=sci_artte xt.

Congreso Mexicano de Investigación Educativa. (2002). Conferencia magistral presentada en el VI Congreso Nacional de Investigación Educativa. Extraído el 10 de diciembre de 2011 desde http://redie.uabc.mx/vol4no1/contenido-amestoy.html

Dilts, R. (2008). El anclaje. Recuperado el 10 de diciembre de 2011 desde http://www.pnl-hipnosis.com/artlanclaje.htm.

Escalante, E., Cervantes, Y., Pernet, J., Piedrahíta, E. (2009). Concepciones docentes sobre la promoción del desarrollo de la destreza "observar" y su evidencia en la práctica pedagógica. Zona próxima $\mathrm{N}^{\mathrm{0}}$ 11. Consultado el de 10 de agosto 2010 desde: http://ciruelo.uninorte.edu.co/pdf/zona_proxima/11/4_Concepciones\%20docentes.pdf.

Justo de la Rosa, M. (2009). Habilidades básicas del pensamiento y competencia para aprender a aprender. Asociación Mundial de Educadores Infantiles (AMEI), "Fortaleciendo las Competencias: Nuevas estrategias, nuevos aprendizajes" Congreso llevado a cabo en Madrid España.

Piaget, J. (1978). La representación del mundo en el niño. Madrid: Morata

Piaget, J. (1986). The Essential Piaget. New York. Basic Books. 
Revolución Educativa (2002). Santa Fe de Bogotá: Ministerio de educación Nacional.

Suarez, J. R., Cabrera, K., Roa, A., \& Guzmán, J. (2007). La formación básica en la Universidad del Norte. Trabajo de grado de Maestría publicado. Universidad del Norte. Barranquilla.

Tapia, V. \& Luna, J. (2010). "Validación de una prueba de habilidades de pensamiento para alumnos de cuarto y quinto de secundaria y primer año de universidad". Lima, Perú. Revista de Investigación en Psicología. Vol. 13, No. 10.

Vigotsky, L. (1978). Mind in society: The development of higher psycological processes. Cambridge: Harvard University Press.

Vigotsky, L. (1987). Pensamiento y lenguaje. Buenos Aires: La Pléyade.

Villarini, A. (1991). Manual para la enseñanza de destrezas de pensamiento. Puerto Rico: OFDP.

Villarini. (1991). La enseñanza orientada al desarrollo del pensamiento. Puerto Rico: PELL.

Villarini. (1997). El currículo orientado al Desarrollo humano Integral. Puerto Rico: OFDP.

Villarini. (2001). El currículo orientado al desarrollo humano integral y el aprendizaje auténtico. Puerto Rico: OFDP.

Villarini. (2001). Teoría y pedagogía del pensamiento sistemático y crítico. Universidad de Puerto Rico. Puerto Rico: OFDP. 